Canad. Math. Bull. Vol. 31 (3), 1988

\title{
SOME CONSEQUENCES OF LAŠNNEV'S THEOREM IN SHAPE THEORY
}

\author{
BY \\ M. ALONSO MORON
}

\begin{abstract}
In this paper we use the Lašnev Theorem in order to give some properties of a class of metrizable spaces having compact metric shape.
\end{abstract}

Introduction and basic notations. In this brief note we deal with metrizable spaces which have the same shape, in the sense of Fox [5] like in [1] and [2], as a compact metrizable space. No special constructions are made; on the contrary, the results in this paper are immediate consequences of the results in [2] and the Lašnev's Theorem, but they maintain the initial geometrical character of shape theory. A more exhaustive research paper on spaces having compact metric shape can be found in [7].

Let us recall some basic concepts from [1] and [2]: If $X$ is a metrizable space, then the space of quasicomponents of $X$ (denoted by $\Delta X$ ) is the space whose elements are the quasicomponents of $X$ and its topology is such that the natural projection $p: X \rightarrow \Delta X$ is a quotient map. If in addition $p$ is a closed map, then we say that the decomposition of $X$ into quasicomponents is upper semicontinuous (denoted by $X \in U S D Q$ ).

As usual $\square(X)$ denotes the space of components of $X$.

We say that a metrizable space belongs to the class $S_{0}\left(X \in S_{0}\right)$ if the two following conditions are satisfied:

(I) $X \in U S D Q$.

(II) (covering) $\operatorname{dim}(\Delta X)=0$.

As we have pointed out in [1] and [2], the class $S_{0}$ behaves well in Fox shape theory in the sense that some results from the compact case can be transferred to the $S_{0}$ case. On the other hand, the following relations hold: (all spaces considered are metrizable)

(I) If $\operatorname{dim}(X)=0$, then $X \in S_{0}$.

(II) If $X$ is a locally compact space with compact components, then $X \in S_{0}$.

(III) In the realm of locally compact spaces the two following statements

Received by the editors November 5, 1986, and, in revised form, May 1, 1987.

Key words: Compact metric shape, Class $S_{0}$, Space of components.

AMS Subject Classification (1980): Primary 54F43; Secondary 54B15.

(c) Canadian Mathematical Society 1987. 
are equivalent:

(a) $X \in U S D Q$

(b) $X \in S_{0}$.

(IV) Locally connected spaces and $A W N R$-spaces are in the class $S_{0}$.

(V) The class $S_{0}$ is closed under mutational retractions (see Lemma 3.3 in [2] ).

(VI) If $X \in S_{0}$, then every quasicomponent is connected, i.e. $\square(X)=\Delta X$ (see Proposition 1.4 in [1] ).

In order to end this introduction, we are going to state Lašnev's result which we shall use in this paper:

TheOREM (see [6] and [4] ). Let $X$ be a metrizable space and $f: X \rightarrow Y$ a closed continuous function onto $Y$, then

$$
Y=Y_{0} \cup\left(\bigcup_{n=1}^{\infty} Y_{n}\right)
$$

where $f^{-1}(y)$ is compact for every $y \in Y_{0}$ and $Y_{n}$ is closed and discrete in $Y$ for $n \geqq 1$.

1. Concerning spaces in the class $S_{0}$ with compact metric shape. We begin this section with the following fact:

Proposition 1. Let $X \in U S D Q$ be a metrizable space without compact quasicomponents, then $X \in S_{0}$.

Proof. By the hypothesis we have that $p: X \rightarrow \Delta X$ is a closed map and from Lašnev's Theorem it follows that $\Delta X=\cup_{n=1}^{\infty} C_{n}$, where $C_{n}$ is closed and discrete for every $n \in N$. Finally from the Countable Sum Theorem for dimension, it follows that $\operatorname{dim}(\Delta X)=0$ and consequently $X \in S_{0}$. In particular every quasicomponent of $X$ is connected.

Example 2. There exist spaces satisfying the hypothesis of Proposition 1 and such that the corresponding space of components is not discrete. For example, let us consider

$$
X=\bigcup_{n=1}^{\infty}(-1 / 2,1 / 2) \times\{1 / n\} \cup \cup(-1,1) \times\{0\}
$$

as a subspace of $R^{2}$.

As a direct consequence of Proposition 1 in this paper and Corollary 2.8 in [2], we have:

COROllary 3. Let $X, Y$ be two spaces satisfying the hypothesis of Proposition 1. If in addition $\operatorname{Sh}(X)=S h(Y)$, then there exists a homeomorphism $\Lambda$ of $\square(X)$ 
onto $\square(Y)$ such that for every $H \subset \square(X)$ with $H=F \cap A$, where $F$ is open and $A$ is closed in $\square(X)$, the equality $\operatorname{Sh}\left(p^{-1}(H)\right)=\operatorname{Sh}\left(q^{-1}(\Lambda(H))\right)$ holds. Where $p: X$ $\rightarrow \square(X), q: Y \rightarrow \square(Y)$ are the corresponding projections.

Let us prove now what we consider as the most significant result in this paper.

Proposition 4. Let $X \in S_{0}$ be a metrizable space with compact metric shape, then every component of $X$ has compact boundary in $X$, the cardinal of the set of all non compact components of $X$ is at most $\aleph_{0}$ and every component has the shape of a metric continuum.

Proof. Let $Y$ be a compact metric space such that $S h(\mathrm{X})=S h(Y)$. From Corollary 1.5 in [2] it follows that there exists a homeomorphism $\Lambda$ of $\square(X)$ onto $\square(Y)$ such that $\operatorname{Sh}\left(X_{0}\right)=\operatorname{Sh}\left(\Lambda\left(X_{0}\right)\right)$ for every component $X_{0}$ of $X$ and consequently every component of $X$ has the shape of a metric continuum. On the other hand, since $\square(Y)$ is a compact metric space we have that $\square(X)$ is a compact metric space and since $p: X \rightarrow \square(X)$ is a closed map we have, see for example Theorem 3.1 in [3], that the boundary of every component is a compact subset of $X$. Finally let $C$ be the subset of all non compact components of $X$. Using the Lašnev's Theorem we have that

$$
\square(X)=F_{0} \cup\left(\bigcup_{n=1}^{\infty} F_{n}\right)
$$

where $p^{-1}(f)$ is compact for every $f \in F_{0}$ and $F_{n}$ is closed and discrete in $\square(X)$ for every $n \in N$. From the compactness of $\square(X)$ it follows that $F_{n}$ is a finite subset for every $n \in N$. On the other hand $C \subset \cup_{n=1}^{\infty} F_{n}$ and consequently

$$
\operatorname{Card}(C) \leqq \operatorname{Card}\left(\bigcup_{n=1}^{\infty} F_{n}\right) \leqq \aleph_{0} .
$$

COROllary 5. Let $X \in U S D Q$ be a metrizable space without compact quasicomponents. If $X$ has compact metric shape then $\operatorname{Card}(\square(X)) \leqq \boldsymbol{\aleph}_{0}$.

REMARK 6. (I) The hypothesis $X \in U S D Q$ is essential in Corollary 5. For example the space $Z=R \times C$ (where $R$ is the real line and $C$ is the cantor set) has the shape of $C$.

(II) The space $X$ described in Example 2 satisfies the hypothesis of Corollary 5.

In order to end, we have the following fact:

Proposition 7. Let $X$ be a metrizable space, then the two following statements are equivalent:

(I) $X$ is compact.

(II) $X \in S_{0}$, all components of $X$ are compact spaces and $X$ has compact metric shape. 


\section{REFERENCES}

1. M. Alonso Moron, Upper semicontinuous decompositions and movability in metric spaces. To appear in Bull. Acad. Polon. Sci.

2. - On the problem of components in shape theory for metrizable spaces. Preprint.

3. D. Burke, Closed mapping, Surveys in General Topology, Academic Press (1980), pp. 1-32.

4. J. Chaber, Generalizations of Lasnev's Theorem, Fund. Math. 119 (1983), pp. 85-91.

5. R. H. Fox, On shape, Fund. Math. 74 (1973), pp. 47-71.

6. N. Lašnev, Continuous decompositions, and closed mappings of metric spaces, Soviet Math. Dokl. 6 (1965), pp. 1504-1506.

7. T. Watanabe, On spaces which have the shape of compact metric spaces, Fund. Math. 104 (1979), pp. 1-11.

Departamento de Matematicas

E.T.S. de Ingenieros de Montes Universidad Politecnica de Madrid

Ciudad Universitaria, Madrid 28040 